\title{
Maxwell wave packets in de Sitter expanding universe
}

\author{
Ion I. Cotăescu ${ }^{\mathrm{a}} \mathbb{0}$, Ion Cotăescu Jr. \\ West University of Timişoara, V. Pârvan Ave. 4, 300223 Timisoara, Romania
}

Received: 18 March 2021 / Accepted: 18 July 2021 / Published online: 29 July 2021

(C) The Author(s) 2021

\begin{abstract}
We study for the first time the propagation of the packets of plane waves of the Maxwell free field in the de Sitter expanding universe as detected by an observer staying at rest in his proper frame with physical de Sitter-Painlevé coordinates. This observes an accelerate propagation of the wave packet along to a null geodesic, laying out a severe exponential decay and a moderate dispersion, increasing exponentially in time during propagation. The example we give is the usual anisotropic Gaussian packet for which we present a short graphical analysis pointing out the accelerated propagation, decay and dispersion. Moreover, we show that the observer perceives his horizon as a mirror stopping the wave packets prepared on it and reflecting those prepared beyond it.
\end{abstract}

\section{Introduction}

The light emitted by different cosmic objects is an important source of empirical data which encapsulate information about the cosmic expansion and possible peculiar velocities of the observed objects. Many studies are devoted to the black hole shadows of various black holes in different flat or expanding universes [1-30]. In contrast, the theory of redshift $[31,32]$ remains at the level of Lemaire's equation $[33,34]$ proposed a long time ago for explaining the Hubble law [35]. When the source has a peculiar velocity, the kinetic corrections due to the Doppler effect are considered as in special relativity [31]. Recently we proposed an improvement of this approach replacing special relativity by our de Sitter relativity [36,37], obtaining a rdshift formula having a new non-trivial term combining the cosmological and kinetic effects [38]. Moreover, we related the black hole shadow and redshift for the Schwarzschild [39] and Reissner-Nordstrom [40] black holes moving with arbitrary peculiar velocities in the de Sitter expanding universe.

a e-mail: icotaescu@yahoo.com (corresponding author)
In all these models the photons are considered as classical massless particles moving along null geodesics since we do not have yet a complete quantum theory of light propagating in curved space-times, despite much progress in studying the Maxwell equations in different manifolds including the de Sitter one. On such space-times of various dimensions, the Maxwell equations were studied either in local charts (called here frames) with static coordinates [41-45] or in comoving frames $[46,47]$. Other studies were devoted to the Maxwell field involved in the cosmological particle creation in Friedmann-Lemaître-Robertson-Walker (FLRW) spacetimes [48-57].

A particular case of FLRW space-time is the expanding portion of the $(1+3)$-dimensional de Sitter manifold known as the de Sitter expanding universe. Based on previous results concerning the de Sitter conserved quantities [59-61], the quantization of the free Maxwell field [46] and the whole QED [58] in the de Sitter expanding universe was performed applying the method of the canonical quantization in radiation, or Coulomb, gauge, well known from the flat case [63]. Recently, one of us proposed the quantum theory of redshift [62] focusing on the expectation values of the conserved one-particle operators but ignoring the propagation. For this reason we devote the present paper to the propagation of the Maxwell wave packets in the de Sitter expanding universe, thus completing the theory of the Maxwell free field canonically quantized in this background.

The starting point is the conformal covariance of the Maxwell equations allowing us to construct an electrodynamics in the de Sitter comoving frames with conformal coordinates taking over all the well-known results of the relativistic electrodynamics in Minkowski space-time [46]. The only problem here is that the Lorentz condition is no longer conformally covariant in any gauge having this property only in the radiation gauge. This means that we must use exclusively this gauge when we transfer the results from the flat case in the conformal frames of the de Sitter expanding universe. Moreover, as mentioned, in this gauge we have the 
opportunity of performing the quantization of the Maxwell free field in canonical manner, as in special relativity [63]. Note that by using this method we do not affect the general gauge covariance of the whole theory which helps one to restore the mentioned gauge after isometries. The principal difference is that, instead of the Poincare isometry group of special relativity, we have now $\mathrm{SO}(1,4)$ isometries whose generators become the one-particle operators of the quantum theory [46] with specific physical meaning [58,61].

In the de Sitter expanding universe there are physical frames with de Sitter-Painlevé coordinates [64,65], formed by the proper, or cosmic, time and physical space coordinates giving the physical distances measured by observers staying in the origins of these frames. The physical coordinates may be related to the conformal ones through transformations depending on time which may change the time evolution picture of the quantum theory [66-68]. For avoiding this difficulty, we set the initial conditions at the time $t_{0}$ when the scale factor of the expanding portion satisfies $a\left(t_{0}\right)=1$ since then the physical and conformal space coordinates coincide [62]. In this approach we may use conformal coordinates for describing the prepared packets but physical ones in observer's proper frames where the wave packets are detected.

In what follows we focus on the propagation of the packets of plane waves of the Maxwell free field in the de Sitter expanding universe paying attention to a pair of sensitive technical problems which are crucial in this approach. The first one is related to the momentum-dependent phase of the plane wave solutions of the Maxwell equations which must depend explicitly on the initial conditions in order to ensure the correct flat limit of our approach [62]. The second problem is related to the detector measuring the wave packet which has to select only the radiation emitted by a remote source. For doing so we assume that the detector filters the momenta along a given direction determining the properties of the detected one-dimensional wave packet [62].

The results we obtain in observer's proper frames with physical coordinates show that the packets of plane waves propagate accelerated, their maximum intensities following null geodesics. Moreover, these have an exponential decay during propagation such that the ratio of the emitted and detected maximum intensities depends on the redshift $z$ as $(1+z)^{4}$. A moderate dispersion of the wave packets can also be derived analytically and pointed out by a graphical analysis. In addition, the horizon of the observer detecting the wave packet is perceived as a mirror stopping the wave packets prepared on it and reflecting those prepared beyond it.

We start in the second section with the canonical quantization of the Maxwell field in the radiation gauge giving the mode expansion in terms of plane wave solutions of the momentum-helicity basis. Furthermore, we define the
Maxwell wave packets in conformal frames showing how these can be detected filtering one-dimensional wave packets. The next section is devoted to the measurement of these wave packets in observer's proper frames with physical coordinates where we derive their field strength, stress energy tensor and intensity. The example we give is of the genuine anisotropic Gaussian packet in momentum representation for which we perform a brief graphical analysis. Finally, we present some concluding remarks.

Here we use the natural Planck units with $c=\hbar=G=1$ and the notations of Refs. [46,62], denoting by $\omega_{H}=\sqrt{\frac{\Lambda}{3}} c$ the de Sitter Hubble constant (frequency). The Hubble time $t_{H}=\frac{1}{\omega_{H}}$ and the Hubble length $l_{H}=\frac{c}{\omega_{H}}$ will have the same form in these units.

\section{Wave packets in conformal frames}

The $(1+3)$-dimensional de Sitter manifold, $M=M_{+} \cup M_{-}$, is a hyperboloid embedded in a $(1+4)$-dimensional pseudoEuclidean manifold that can be covered by two local charts interpreted as the expanding $\left(M_{+}\right)$and, respectively, collapsing $\left(M_{-}\right)$spatially flat FLRW universes [69]. In what follows we consider the comoving frames of the expanding portion, $M_{+}$, equipped with two sets of local coordinates, i.e. the conformal pseudo-Euclidean ones, $\left\{t_{c}, \mathbf{x}_{c}\right\}$, and the physical de Sitter-Painlevé coordinates, $\{t, \mathbf{x}\}$. In the conformal frame we have the conformal time $t_{c}<0$ and the conformal Cartesian spaces coordinates $x_{c}^{i}(i, j, k, \ldots=1,2,3)$, known as the comoving space coordinates [69], giving the line element

$\mathrm{d} s^{2}=g_{\mu \nu}\left(x_{c}\right) \mathrm{d} x_{c}^{\mu} \mathrm{d} x_{c}^{\nu}=\frac{1}{\omega_{H}^{2} t_{c}^{2}}\left(\mathrm{~d} t_{c}{ }^{2}-d \mathbf{x}_{c} \cdot d \mathbf{x}_{c}\right)$.

The de Sitter-Painlevé coordinates [64] on $M_{+}$can be introduced directly by substituting

$t_{c}=-\frac{1}{\omega_{H}} \mathrm{e}^{-\omega_{H} t}, \quad \mathbf{x}_{c}=\mathbf{x e}^{-\omega_{H} t}$,

where $t \in(-\infty, \infty)$ is the proper, or cosmic, time, while the $x^{i}$ are the physical Cartesian space coordinates of an observer staying at rest in origin. In this frame the line element

$$
\begin{aligned}
\mathrm{d} s^{2} & =g_{\mu \nu}(x) \mathrm{d} x^{\mu} \mathrm{d} x^{\nu} \\
& =\left(1-\omega_{H}^{2} \mathbf{x}^{2}\right) \mathrm{d} t^{2}+2 \omega_{H} \mathbf{x} \cdot \mathrm{d} \mathbf{x} \mathrm{d} t-\mathrm{d} \mathbf{x} \cdot \mathrm{d} \mathbf{x}
\end{aligned}
$$

points out the observer's horizon at $\omega_{H}^{-1}$ such that the condition $|\mathbf{x}|<\omega_{H}^{-1}$ is mandatory for the positions that can be observed. 
In the frames with combined coordinates, $\left\{t, \mathbf{x}_{c}\right\}$, the metric takes the FLRW form

$\mathrm{d} s^{2}=\mathrm{d} t^{2}-a(t)^{2} \mathrm{~d} \mathbf{x}_{c} \cdot \mathrm{d} \mathbf{x}_{c}, \quad a(t)=e^{\omega_{H} t}$,

where $a(t)$ is the scale factor of the expanding portion $M_{+}$ which can be rewritten in the conformal frame,

$a\left(t_{c}\right) \equiv a\left[t\left(t_{c}\right)\right]=-\frac{1}{\omega_{H} t_{c}}$,

as a function defined for $t_{c}<0$.

\subsection{Maxwell wave packets}

The cornerstone of our approach is the conformal covariance of the Maxwell equations allowing us to take over all the results of special relativity in the comoving frames with conformal coordinates, $\left\{t_{c}, \mathbf{x}_{c}\right\}$, of the de Sitter expanding universe [46]. Moreover, for imposing the Lorentz condition, which is not conformally covariant, we must choose an electromagnetic potential $A_{\mu}^{c}$ in the radiation gauge,

$A_{0}^{c}=0, \quad \partial_{x_{c}^{i}} A_{i}^{c}=0$,

in which the Maxwell equation takes the same form as in the flat case,

$\left(\partial_{t_{c}}^{2}-\Delta_{c}\right) A_{i}^{c}=0$.

Then the corresponding quantum field, $\mathcal{A}_{i}^{c}$, can be expressed in terms of plane wave solutions as [46]

$$
\begin{aligned}
\mathcal{A}_{i}^{c}\left(x_{c}\right)= & \mathcal{A}_{i}^{c(+)}\left(x_{c}\right)+\mathcal{A}_{i}^{c(-)}\left(x_{c}\right) \\
= & \int \mathrm{d}^{3} k \sum_{\lambda}\left[e_{i}\left(\mathbf{n}_{k}, \lambda\right) f_{\mathbf{k}}\left(x_{c}\right) a(\mathbf{k}, \lambda)\right. \\
& \left.+\left[e_{i}\left(\mathbf{n}_{k}, \lambda\right) f_{\mathbf{k}}\left(x_{c}\right)\right]^{*} a^{\dagger}(\mathbf{k}, \lambda)\right],
\end{aligned}
$$

where $a(\mathbf{k}, \lambda)$ are the field operators in momentum representation, $e_{i}\left(\mathbf{n}_{k}, \lambda\right)$ are the polarization vectors while

$f_{\mathbf{k}}\left(x_{c}\right)=\frac{1}{(2 \pi)^{3 / 2}} \frac{1}{\sqrt{2 k}} e^{i \delta(\mathbf{k})-i k t_{c}+i \mathbf{k} \cdot \mathbf{x}_{c}}$,

are the fundamental solutions of the d'Alambert equation (7) depending on the momenta $\mathbf{k}=k \mathbf{n}_{k}$ (with $k=|\mathbf{k}|$ ). The phase

$\delta(\mathbf{k})=k t_{c 0}-\mathbf{k} \cdot \mathbf{x}_{c 0}$

is given by the initial condition showing that the field was prepared in $\mathbf{x}_{c 0}$ at the time $t_{c 0}$. Thus we obtain the same solution as in the flat case but with a new momentum-dependent phase which cannot be ignored as long as the energy operator on $M_{+}$depends on its form [46,62].

The functions $f_{\mathbf{k}}\left(x_{c}\right)$, assumed to be of positive frequencies, and those of negative frequencies, $f_{\mathbf{k}}(x)^{*}$, satisfy the orthonormalization relations [46]

$$
\begin{aligned}
& \left(f_{\mathbf{k}}, f_{\mathbf{k}^{\prime}}\right)=-\left(f_{\mathbf{k}}^{*}, f_{\mathbf{k}^{\prime}}^{*}\right)=\delta^{3}\left(\mathbf{k}-\mathbf{k}^{\prime}\right), \\
& \left(f_{\mathbf{k}}, f_{\mathbf{k}^{\prime}}^{*}\right)=0,
\end{aligned}
$$

with respect to the Hermitian form

$(f, g)=i \int \mathrm{d}^{3} x_{c} f^{*}\left(t_{c}, \mathbf{x}_{c}\right) \stackrel{\leftrightarrow}{\partial_{t_{c}}} g\left(t_{c}, \mathbf{x}_{c}\right)$,

where we denote $f \stackrel{\leftrightarrow}{\partial} g=f \partial g-g \partial f$.

The polarization vectors $\mathbf{e}\left(\mathbf{n}_{k}, \lambda\right)$ in the gauge (6) must be orthogonal to the momentum direction,

$\mathbf{k} \cdot \mathbf{e}\left(\mathbf{n}_{k}, \lambda\right)=0$,

for any polarization $\lambda= \pm 1$. We remind the reader that the polarization can be defined in different manners independent of the form of the scalar solutions $f_{\mathbf{k}}$. In general, the polarization vectors have c-number components which must satisfy [70]

$$
\begin{aligned}
\mathbf{e}\left(\mathbf{n}_{k}, \lambda\right) \cdot \mathbf{e}\left(\mathbf{n}_{k}, \lambda^{\prime}\right)^{*} & =\delta_{\lambda \lambda^{\prime}}, \\
\sum_{\lambda} e_{i}\left(\mathbf{n}_{k}, \lambda\right) e_{j}\left(\mathbf{n}_{k}, \lambda\right)^{*} & =\delta_{i j}-\frac{k^{i} k^{j}}{k^{2}} .
\end{aligned}
$$

Under such circumstances we can perform the canonical quantization assuming that the field operators fulfil the standard commutation relations from which the non-vanishing ones are $[46,62]$

$\left[a(\mathbf{k}, \lambda), a^{\dagger}\left(\mathbf{k}^{\prime}, \lambda^{\prime}\right)\right]=\delta_{\lambda \lambda^{\prime}} \delta^{3}\left(\mathbf{k}-\mathbf{k}^{\prime}\right)$.

Moreover, we consider a unique vacuum state, $|0\rangle$, of the Fock space such that

$a(\mathbf{k}, \lambda)|0\rangle=0, \quad\langle 0| a^{\dagger}(\mathbf{k}, \lambda)=0$.

Then the sectors with a given number of particles may be constructed using the standard methods for obtaining the generalized momentum-helicity basis of the Fock space. Thus we can say that in the de Sitter expanding universe the canonical quantization of the free Maxwell field can be done in the gauge (6) just as in Minkowski space-time [63] where this simple method prevents one of using the Gupta-Bleuler formalism [71,72]. Moreover, as in the flat case, we can verify that this method is compatible with the covariance under the $S O(1,4)$ isometries since after each isometry we can perform a suitable gauge transformation for restoring the mentioned gauge. 
A simple model which save us from complicated calculations is that of the one-particle wave packets. In our Heisenberg picture these are given by the time-independent oneparticle states,

$|\alpha\rangle=\int \mathrm{d}^{3} k \sum_{\lambda} \alpha_{\lambda}(\mathbf{k}) a^{\dagger}(\mathbf{k}, \lambda)|0\rangle$,

defined by the square integrable functions in momentum representation $\alpha_{\lambda}(\mathbf{k})$ which must satisfy the normalization condition

$\langle\alpha \mid \alpha\rangle=\int \mathrm{d}^{3} k \sum_{\lambda}\left|\alpha_{\lambda}(\mathbf{k})\right|^{2}=1$.

The corresponding 'wave functions'

$$
\begin{aligned}
A_{i}^{c}\left(x_{c}\right) & =\left\langle 0\left|\mathcal{A}_{i}^{c}\left(x_{c}\right)\right| \alpha\right\rangle \\
& =\int \mathrm{d}^{3} k \sum_{\lambda} e_{i}\left(\mathbf{n}_{k}, \lambda\right) f_{\mathbf{k}}\left(x_{c}\right) \alpha_{\lambda}(\mathbf{k})
\end{aligned}
$$

define normalized wave packets having the norm

$\left\|A^{c}\right\|^{2}=\delta_{i j}\left(A_{i}^{c}, A_{j}^{c}\right)=\int \mathrm{d}^{3} k \sum_{\lambda}\left|\alpha_{\lambda}(\mathbf{k})\right|^{2}=1$,

resulting from Eqs. (11) and (12).

The expectation values of the one-particle operators in the state $|\alpha\rangle$ can be calculated simply as

$\langle\alpha|\mathcal{X}| \alpha\rangle=\delta_{i j}\left(A_{i}^{c}, \hat{X} A_{j}^{c}\right)$

avoiding the tedious algebra of field operators. For example for the momentum operators $\hat{P}^{i}=-i \partial_{x_{c}^{i}}$ we may write,

$\delta_{i j}\left(A_{i}^{c},\left(\hat{P}^{i}\right)^{n} A_{j}^{c}\right)=\int \mathrm{d}^{3} k\left(k^{i}\right)^{n} \sum_{\lambda} \alpha_{\lambda}(\mathbf{k})^{2}$,

and similarly for the Pauli-Lubanski operator [62]. The energy operator has a more complicated form, depending on the phase of the functions $\alpha_{\lambda}(\mathbf{k})[46,62]$.

\subsection{Preparing and detecting wave packets}

Let us analyze how an observer $O$ measures in his proper frame $\left\{t_{c}, \mathbf{x}_{c}\right\}_{O}$ a wave packet prepared by another observer $O^{\prime}$ in his frame $\left\{t_{c}, \mathbf{x}_{c}^{\prime}\right\}_{O^{\prime}}$. We simplify the geometry by choosing the space axes of these two frames parallel with the orthonormal frame $\left\{\mathbf{e}_{1}, \mathbf{e}_{2}, \mathbf{e}_{3}\right\}$ such that the origin $O^{\prime}$ is translated with respect to $O$, as

$\mathbf{x}_{c}^{\prime}=\mathbf{x}_{c}+\mathbf{d}$

with the translation parameter $\mathbf{d}=\mathbf{e}_{3} d$ which has the direction $O^{\prime} O$.
In this set-up we assume that the observer $O^{\prime}$ prepares the state $|\alpha\rangle$ in his proper comoving frame at the initial time

$t_{c 0}=-\frac{1}{\omega_{H}} \rightarrow t_{0}=0$,

when $a=1$ and, consequently, the conformal and physical space coordinates coincide. This choice guarantees that the expectation values of the conserved operators calculated at this time in the conformal frame keep their forms in the physical frame.

As the packet is prepared in the origin of the frame $\left\{t_{c}^{\prime}, \mathbf{x}_{c}^{\prime}\right\}_{O^{\prime}}$ at the time (26) the phase (10) takes the form

$\delta^{\prime}(\mathbf{k})=-\frac{k}{\omega_{H}}$.

Then the wave packet

$$
\begin{aligned}
A_{i}^{c \prime}\left(x_{c}\right)= & \frac{1}{(2 \pi)^{3 / 2}} \int \frac{\mathrm{d}^{3} k}{\sqrt{2 k}} e^{-i k\left(t_{c}+\frac{1}{\omega_{H}}\right)+i \mathbf{k} \cdot \mathbf{x}_{c}} \\
& \times \sum_{\lambda} e_{i}\left(\mathbf{n}_{k}, \lambda\right) \alpha_{\lambda}(\mathbf{k})
\end{aligned}
$$

has a correct flat limit since $\lim _{\omega_{M} \rightarrow 0}\left(t_{c}+\frac{1}{\omega_{H}}\right)=t$. Note that we meet similar properties in the case of the rest frame vacua of the massive fields we proposed recently [73-76], which fix suitable phases ensuring correct flat limits.

In applications it is convenient to introduce the polarization angle $\sigma(\mathbf{k})$ substituting

$$
\begin{aligned}
\alpha_{\lambda=1}(\mathbf{k}) & =\alpha(\mathbf{k}) \cos \sigma\left(\mathbf{n}_{k}\right), \\
\alpha_{\lambda=-1}(\mathbf{k}) & =\alpha(\mathbf{k}) \sin \sigma\left(\mathbf{n}_{k}\right),
\end{aligned}
$$

taking into account that the new real valued function $\alpha(\mathbf{k})$ is normalized,

$\int \mathrm{d}^{3} k \alpha(\mathbf{k})^{2}=1$

Thus we can say that any wave packet is determined by two scalar functions $\alpha(\mathbf{k})$ and $\sigma\left(\mathbf{n}_{k}\right)$.

Once the wave packet is prepared this evolves causally until an ideal apparatus measures some of its parameters [77]. The detector of $O$ must select only the photons coming from the source $O^{\prime}$ whose momenta are parallel with $\mathbf{e}_{3}$. This means that the domain of momenta measured by $O$ is [62]

$$
\Delta=\left\{\mathbf{k} \mid-\frac{\Delta k}{2} \leq k^{1} \leq \frac{\Delta k}{2}, \quad-\frac{\Delta k}{2} \leq k^{2} \leq \frac{\Delta k}{2}, k^{3}>0\right\}
$$


where $\Delta k$ is a small quantity. Then we may evaluate the integrals over $\Delta$ as.

$$
\begin{aligned}
\int_{\Delta} d^{3} k F(\mathbf{k}) & =\int_{-\frac{\Delta k}{2}}^{\frac{\Delta k}{2}} \mathrm{~d} k^{1} \int_{-\frac{\Delta k}{2}}^{\frac{\Delta k}{2}} \mathrm{~d} k^{2} \int_{-\infty}^{0} \mathrm{~d} k^{3} F\left(k^{1}, k^{2}, k^{3}\right) \\
& \simeq(\Delta k)^{2} \int_{0}^{\infty} \mathrm{d} k F(0,0, k)
\end{aligned}
$$

according to the mean value theorem.

Under such circumstances, the observer $O$ filters the onedimensional packet [62]

$$
A_{i}^{c}\left(t_{c}, x_{c}^{3}\right)=\frac{1}{\sqrt{\kappa}} \int_{0}^{\infty} \mathrm{d} k \sum_{\lambda} e_{i}(\lambda) \tilde{f}_{\mathbf{k}}\left(t_{c}, x_{c}^{3}\right) \alpha_{\lambda}(0,0, k)
$$

along the third axis. The constant

$\kappa=\int_{0}^{\infty} \mathrm{d} k \sum_{\lambda} \alpha_{\lambda}(0,0, k)^{2}$.

ensures the normalization since the functions $\alpha(0,0, k)$ are no longer normalized. The new functions

$\tilde{f}_{\mathbf{k}}\left(t_{c}, x_{c}^{3}\right)=\frac{1}{\sqrt{2 \pi}} \frac{1}{\sqrt{2 k}} \mathrm{e}^{i \delta(\mathbf{k})-i k t_{c}+i k x_{c}^{3}}$,

are orthonormal with respect to the new Hermitian form

$$
(\tilde{f} ; \tilde{g})=i \int \mathrm{d} x_{c} \tilde{f}^{*}\left(t_{c}, x_{c}^{3}\right) \stackrel{\leftrightarrow}{\partial_{t_{c}}} \tilde{g}\left(t_{c}, x_{c}^{3}\right) .
$$

As in our experiment we select only the momenta oriented along $\mathbf{e}_{3}$, the polarizations vectors $\mathbf{e}( \pm 1)=\frac{1}{\sqrt{2}}\left(\mathbf{e}_{1} \pm i \mathbf{e}_{2}\right)$ are in the plane $\left\{\mathbf{e}_{1}, \mathbf{e}_{2}\right\}$. Moreover, since here the functions $\alpha_{\lambda}$ are those of Eq. (28) we may use the substitutions (29) and (30), which now read

$$
\begin{aligned}
\alpha_{\lambda=1}(0,0, k) & =\alpha(0,0, k) \cos \sigma, \\
\alpha_{\lambda=-1}(0,0, k) & =\alpha(0,0, k) \sin \sigma,
\end{aligned}
$$

where the functions $\alpha$ satisfy

$$
\int_{0}^{\infty} \mathrm{d} k \alpha(0,0, k)^{2}=\kappa
$$

as it results from Eq. (35). Now $\sigma=\sigma\left(\mathbf{e}_{3}\right)$ denotes the constant polarization angle of the direction $\mathbf{e}_{3}$ giving the polarization unit vector

$\mathbf{e}(\sigma)=\mathbf{e}(1) \cos \sigma+\mathbf{e}(-1) \sin \sigma$.

On the other hand, from the point of view of the observer $O$ the wave packet is prepared at the time (26) in $\mathbf{x}_{c 0}=-\mathbf{d}$ such that the phase (10) becomes [62]

$\delta(\mathbf{k})=-\frac{k}{\omega_{H}}+\mathbf{k} \cdot \mathbf{d}$.

Thus we arrive at the final form of the potential of the onedimensional packet

$A_{i}^{c}\left(t_{c}, x_{c}^{3}\right)=e_{i}(\sigma) A\left(X_{c}\right)$,

having the fixed direction along the unit vector $\mathbf{e}(\sigma)$ and depending on the amplitude

$A\left(X_{c}\right)=\frac{1}{\sqrt{2 \pi \kappa}} \int_{0}^{\infty} \frac{\mathrm{d} k}{\sqrt{2 k}} \mathrm{e}^{-i k X_{c}} \alpha(0,0, k) \in \mathbb{C}$,

where

$X_{c}=t_{c}+\frac{1}{\omega_{H}}-x_{c}^{3}-d$.

This amplitude represents the principal integral we have to solve for defining the wave packet.

\section{Wave packets in physical frames}

Our principal goal is to study how the observer $O$ measures in the origin of his proper frame, $\{t, \mathbf{x}\}_{O}$, the propagation of the one-dimensional wave packet selected from an arbitrary wave packet prepared in the origin of the frame $\{t, \mathbf{x}\}_{O}$. These frames have physical coordinates (2) such that the translation (25) gives the time-dependent translation

$\mathbf{x}^{\prime}=\mathbf{x}+\mathbf{d}(t), \quad \mathbf{d}(t)=\mathbf{d} e^{\omega_{H} t}$,

where $\mathbf{d}$ is now the translation parameter at the initial time $t_{0}=0$. Then we can find that the velocity of $O^{\prime}$ with respect to $O, \mathbf{v}(t)=\dot{\mathbf{d}}(t)=\omega_{H} \mathbf{d}(t)$, complies with the velocity law, which is confused sometimes with the Hubble one [31,32]. Now we understand why the choice of the initial time $t_{0}=0$ when the wave packet is prepared simplifies the parametrization of our set-up.

\subsection{Propagating wave packets}

As the potentials cannot be measured directly we must look for relevant quantities as the wave intensity or the density of energy which must be derived now in the frame $\{t, \mathbf{x}\}_{O}$. For simplicity we consider the plane polarization fixing the polarization angle

$\sigma\left(\mathbf{e}_{3}\right)=\frac{\pi}{4} \rightarrow \mathbf{e}(\sigma)=\mathbf{e}_{1}$, 
such that we are left only with the component $A_{1}^{c}$ of the potential (43). This generates the components in the frame $\{t, \mathbf{x}\}_{O}$,

$A_{0}(t, \mathbf{x})=\frac{\partial x_{c}^{i}}{\partial t} A_{i}^{c}\left(t_{c}, x_{c}^{3}\right)=-\omega_{H} x^{1} \mathrm{e}^{-\omega_{H} t} A(X)$,

$A_{1}(t, \mathbf{x})=\frac{\partial x_{c}^{i}}{\partial x^{1}} A_{i}^{c}\left(t_{c}, x_{c}^{3}\right)=\mathrm{e}^{-\omega_{H} t} A(X)$,

$A_{2}(t, \mathbf{x})=A_{3}(t, \mathbf{x})=0$,

where $A$ is the amplitude (44) depending on the new variable

$X=\frac{1-\mathrm{e}^{-\omega_{H} t}}{\omega_{H}}-x^{3} \mathrm{e}^{-\omega_{H} t}-d$,

obtained from Eq. (45) after the substitution (2). These potentials satisfy the Maxwell equation and the Lorentz condition in the frame $\{t, \mathbf{x}\}_{O}$ where the field strength, $F^{\mu \nu}=$ $g^{\mu \alpha} g^{\nu \beta}\left(\partial_{\alpha} A_{\beta}-\partial_{\beta} A_{\alpha}\right)$, reads

$$
\begin{aligned}
F= & e^{-2 \omega_{H} t} \partial_{X} A(X) \\
& \times\left(\begin{array}{cccc}
0 & 1 & 0 & 0 \\
-1 & 0 & -\omega_{H} x^{2} & -\left(1+\omega_{H} x^{3}\right) \\
0 & \omega_{H} x^{2} & 0 & 0 \\
0 & 1+\omega_{H} x^{3} & 0 & 0
\end{array}\right) .
\end{aligned}
$$

Furthermore, we derive the stress energy tensor components

$T^{\mu \nu}=F_{\cdot \alpha}^{\mu *} F^{\alpha \nu}-\frac{1}{4} g^{\mu \nu} F_{\cdot \alpha}^{\beta *} F_{\cdot \beta}^{\alpha}$,

which can be written as

$$
\begin{aligned}
T & =\mathrm{e}^{-4 \omega_{H} t}\left|\partial_{X} A(X)\right|^{2} \\
& \times\left(\begin{array}{cccc}
1 & \omega_{H} x^{1} & \omega_{H} x^{2} & p \\
\omega_{H} x^{1} & \omega_{H}^{2}\left(x^{1}\right)^{2} & \omega_{H}^{2} x^{1} x^{2} & \omega_{H} x^{1} p \\
\omega_{H} x^{2} & \omega_{H}^{2} x^{1} x^{2} & \omega_{H}^{2}\left(x^{2}\right)^{2} & \omega_{H} x^{2} p \\
p & \omega_{H} x^{1} p & \omega_{H} x^{2} p & p^{2}
\end{array}\right),
\end{aligned}
$$

where $p=1+\omega_{H} x^{3}$. It is not difficult to verify that this satisfies the conservation rule $\nabla_{\mu} T^{\mu \nu}=0$.

Hence we obtained the complete theory of the Maxwell free field in physical frames of $M_{+}$whose physical quantities (50) and (52) remain invariant under the gauge transformations,

$$
A_{\mu}(x) \rightarrow A_{\mu}(x)+\partial_{\mu} \phi(x),
$$

in these frames. Thus the initial restriction imposed by the gauge (6) does not work in physical frames where the potential has the components defined by Eq. (48) up to an arbitrary gauge (53).
For studying the propagation of our wave packet we focus on the intensity which coincides with the density of energy,

$I(t, x)=\delta_{i j} F^{0 i *} F^{0 j} \equiv T^{00}$,

introducing the factorization

$I(t, x)=e^{-4 \omega_{H} t} I_{0}(X), \quad I_{0}(X)=\left|\partial_{X} A(X)\right|^{2}$,

and rewriting in a simpler form, $x^{3} \rightarrow x$. Thus we separate the exponential decay from the function $I_{0}(X)$ depending only on the amplitude (44). In what follows we construct this amplitude by using only positive definite test functions $\alpha(\mathbf{k})$ which guarantee that $\left|\partial_{X} A(X)\right| \leq\left|\partial_{X} A(X)\right|_{X=0}$. Consequently, the function $I_{0}(t, x)$ has an absolute (or global) maximum $I_{m}(t)=e^{-\omega_{H} t} I_{0}(0)$ in the point

$x_{m}(t)=\frac{1}{\omega_{H}}\left[\left(1-\omega_{H} d\right) \mathrm{e}^{\omega_{H} t}-1\right]$,

where $X=0$. Remarkably, this is just the equation of the null geodesics passing through $O^{\prime}$ and $O[78,79]$. Once the wave packet is prepared at $t=0$ in the point $x=x_{m}(0)=-d$, its maximum propagates arriving in $O$ at the time

$t_{f}=-\frac{1}{\omega_{H}} \ln \left(1-\omega_{H} d\right)$,

when $x_{m}\left(t_{f}\right)=0$.

The exponential factor produces the decay of the maximum intensity from $I_{m}(0)=I_{0}(0)$ up to

$I_{m}\left(t_{f}\right)=\left(1-\omega_{H} d\right)^{4} I_{0}(0)$,

which decreases with the distance $d$. Thus we find that the emitted and detected maximum intensities are related through the binomial depending on the redshift $z$. Indeed, according to the Lemaitre equation of Hubble-s law we may write

$1+z=\frac{a\left(t_{f}\right)}{a(0)}=\frac{1}{1-\omega_{H} d} \rightarrow I_{m}\left(t_{f}\right)=\frac{I_{m}(0)}{(1+z)^{4}}$,

showing the opportunity of estimating the emitted maximum intensity, $I_{m}(0)$, in terms of the measured maximum intensity, $I_{m}\left(t_{f}\right)$, and redshift. Similarly, we deduce the distance $O^{\prime} O$ at the moment $t_{f}$ when the wave packet is detected in $O$ reads

$d\left(t_{f}\right)=\frac{d}{1-\omega_{H} d}=(1+z) d$,

as predicted by Lemaitre's equation and recovered explicitly in Refs. [38,39]. 
The space dispersion of the wave packet, $\delta x(t)$, measures the width of the function $I(t, X)$ at a given time. This depends on the width $\delta X$ of the function $I_{0}(X)$, which is a constant quantity depending on the profile of this function. Then, according to Eq. (49), we deduce that the physical dispersion, $\delta x(t)=\delta X e^{\omega_{H} t}$, increases exponentially in time from $\delta x(0)=\delta X$ up to $\delta x\left(t_{f}\right)=(1+z) \delta X$.

The velocity of the wave packet is that of its maximum intensity,

$v_{m}(t)=\dot{x}_{m}(t)=\left(1-\omega_{H} d\right) \mathrm{e}^{\omega_{H} t}\left\{\begin{array}{l}>0 \text { if } d<\omega_{H}^{-1} \\ =0 \text { if } d=\omega_{H}^{-1} \\ <0 \text { if } d>\omega_{H}^{-1}\end{array}\right.$,

resulting from Eq. (56). Hereby we understand that $O$ receives only the packets with $v_{m}(t)>0$, prepared inside the horizon. Otherwise, $O$ perceives the horizon as trapping or reflecting the wave packets prepared on or beyond it. Inside the horizon the propagation is accelerated having the acceleration $a_{m}(t)=\dot{v}_{m}(t)=\omega_{H} v_{m}(t)$, which complies with a Hubble type rule. Consequently, the velocity is increasing from the initial value $v_{m}(0)=1-\omega_{H} d$ up to the speed of light $v_{m}\left(t_{f}\right)=1$ detected by $O$. Note that for the observer $O^{\prime}$ the velocity of the prepared wave packet is also the speed of light, since from his point of view $d^{\prime}=0$.

Thus we described completely the propagation of the detected wave packets whose specific features have to be studied in each particular case separately as in the next example.

\subsection{Anisotropic Gaussian wave packet}

Let us consider now that $O^{\prime}$ prepares the anisotropic Gaussian packet in momentum representation defined by the real valued function

$\alpha(\mathbf{k})=\alpha_{1}\left(k_{1}\right) \alpha_{2}\left(k_{2}\right) \alpha_{3}\left(k_{3}\right)$,

where

$\alpha_{i}\left(k^{i}\right)=\left(\frac{a_{i}^{2}}{\pi}\right)^{\frac{1}{4}} e^{-\frac{a_{i}^{2}}{2}\left(k^{i}-\bar{k}^{i}\right)^{2}}, \quad a_{i}, \bar{k}^{i}>0, \quad i=1,2,3$,

which satisfies the normalization condition (31). Important conserved quantities observed by $O^{\prime}$ are the expectation values of the momentum components

$P^{\prime i}=\int_{0}^{\infty} d k k^{i} \alpha(\mathbf{k})^{2}=\bar{k}^{i}$ and the corresponding dispersions

$\operatorname{disp} P^{\prime i}=\int_{0}^{\infty} d k\left(k^{i}\right)^{2} \alpha(\mathbf{k})^{2}-\left(\bar{k}^{i}\right)^{2}=\frac{1}{2 a_{i}^{2}}$

calculated according to the general rule (24).

The observer $O$ filters the wave packet measuring only the momenta in the domain (32) such that the new wave function $\alpha(0,0, k)$ will give the constant (40) that now reads

$$
\begin{aligned}
\kappa & =\int_{0}^{\infty} d k \alpha(0,0, k)^{2} \\
& =\frac{a_{1} a_{2}}{2 \pi} e^{-a_{1}^{2}\left(\bar{k}^{1}\right)^{2}-a_{2}^{2}\left(\bar{k}^{2}\right)^{2}}\left[1+\operatorname{erf}\left(a_{3} \bar{k}^{3}\right)\right] .
\end{aligned}
$$

With its help we derive the expectation value of the momentum component

$P^{3}=\frac{1}{\kappa} \int_{0}^{\infty} d k k \alpha(0,0, k)^{2}=\bar{k}^{3}+\frac{\beta}{a_{3}}=P^{\prime 3}+\frac{\beta}{a_{3}}$,

where the constant

$\beta=\frac{\mathrm{e}^{-\left(a_{3} \bar{k}^{3}\right)^{2}}}{\sqrt{\pi}\left[1+\operatorname{erf}\left(a_{3} \bar{k}^{3}\right)\right]}$

takes values in the domain $0<\beta \leq \pi^{-\frac{1}{2}}$ as long as $a_{3} \bar{k}^{3}>$ 0 . The corresponding dispersion,

$$
\begin{aligned}
\operatorname{disp} P^{3} & =\frac{1}{\kappa} \int_{0}^{\infty} d k k^{2} \alpha(0,0, k)^{2}-\left(P^{3}\right)^{2} \\
& =\operatorname{disp} P^{\prime 3}\left(1-2 \beta a^{3} \bar{k}^{3}-2 \beta^{2}\right),
\end{aligned}
$$

spans the domain

$$
\left(1-\frac{2}{\pi}\right) \operatorname{disp} P^{\prime 3} \leq \operatorname{disp} P^{3} \leq \operatorname{disp} P^{\prime 3} .
$$

The expectation values of the energies of the emitted and detected photon as observed by $O$ are derived in Ref. [62] where we show that these comply with the Lemaitre rule of Hubble's law.

The space-time behavior of the Gaussian wave packet can be studied since in this case the integral (44) is known. In our notations this can be expressed in terms of modified Bessel functions $K$ as

$A(X)=\sqrt{\frac{\beta}{2 \pi}} \sqrt{\xi} e^{-\xi^{2}} K_{\frac{1}{4}}\left(-\xi^{2}\right)$,

where

$\xi=\frac{1}{2}\left(\frac{X}{a_{3}}+i a_{3} \bar{k}^{3}\right)$

depends on our principal variable $X$, given by Eq. (49). This amplitude is convergent since this behaves as $|\xi|^{-\frac{1}{2}}$ for large 

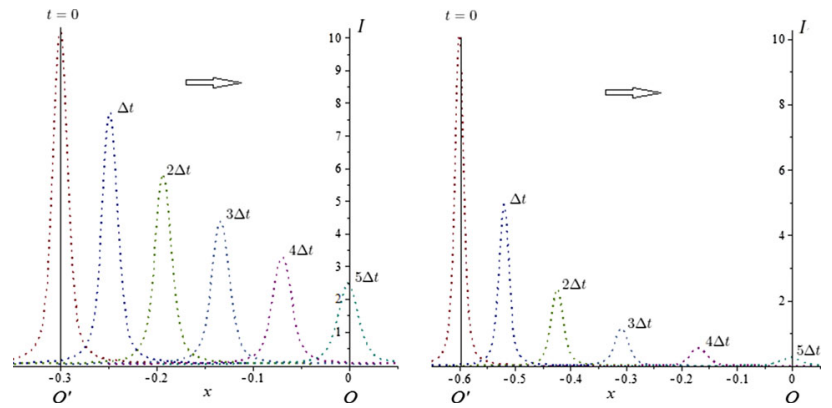

Fig. 1 Profiles of the intensity $I(t, x)$ of the Gaussian wave packet prepared in $O^{\prime}$ (with $a_{3}=0.005$ and $\bar{k}^{3}=100$ ) as observed by $O$ at consecutive epochs. In the left panel $d=0.3$ and $\Delta t=0.0713$, while in the right panel $d=0.6$ and $\Delta t=0.1832$

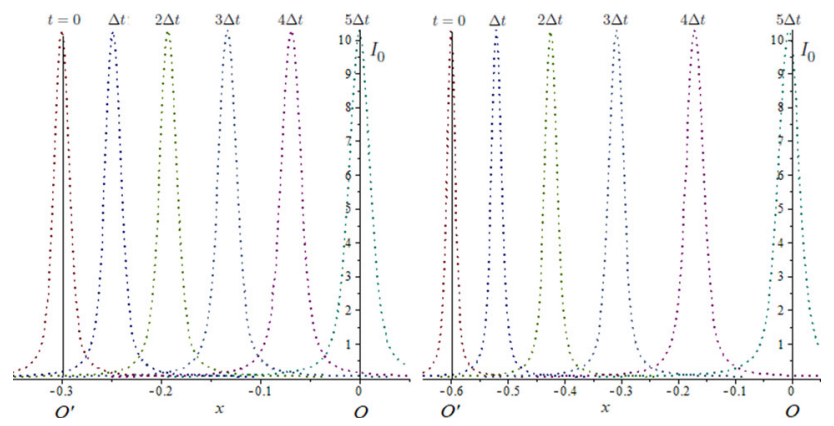

Fig. 2 Space dispersion of the profiles of $I_{0}(t, x)$ of the Gaussian wave packet prepared in $O^{\prime}$ (with $a_{3}=0.005$ and $\bar{k}^{3}=100$ ) as observed by $O$ at consecutive epochs. In the left panel $d=0.3$ and $\Delta t=0.0713$, while in the right panel $d=0.6$ and $\Delta t=0.1832$

values of $|\xi|$ while the singularity in $\xi=0$ of the function $K_{\frac{1}{4}}\left(-\xi^{2}\right)$ is avoided as long as $|\xi| \geq \frac{1}{2} a_{3} \bar{k}^{3}>0$ [80]. With its help we construct the intensity (55) observing that this behaves as $|\xi|^{-3}$ when $|\xi| \rightarrow \infty$. Now we may plot the profiles of these functions at consecutive times for studying how this wave packet evolves.

We perform our graphic analysis in the physical frame $\{t, \mathbf{x}\}_{O}$ by using Hubble units in order to avoid extremely large or small numbers. For the physical distances we use the Hubble length $l_{H}$ such that the horizon is at $|x|=1$. The time is measured in units of Hubble's time $t_{H}$ while for momenta we introduce the unit $p_{H}=\omega_{H}$ (i.e. $\frac{\hbar \omega_{H}}{c}$ in SI units). Thus we obtain intuitive profiles of the wave packets, pointing out their principal features, but far from the physical reality. In Fig. 1 we show how this wave packet propagates when this is prepared at two different distances, $d=0.3$ and, respectively, $d=0.6$. The decay and acceleration are obvious but the dispersion is hidden by this decay. However, we may pint out the dispersion by plotting the function $I_{0}(X)$ as in Fig. 2 where we see that the dispersion is increasing with the propagation time (57). The horizon effects are illustrated in Fig. 3.

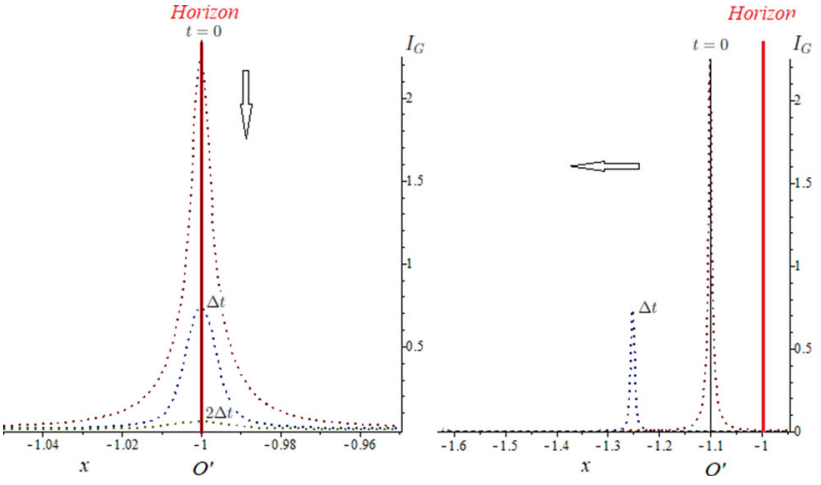

Fig. 3 Horizon effects of the Gaussian wave packet with $a_{3}=0.009$ and $\bar{k}^{3}=100$ plotted at consecutive epochs with $\Delta t=0.9197$. In the left panel the intensity of the wave packet prepared on horizon remains static while the packet prepared beyond the horizon appears as being reflected

Finally, we observe that the packet filtered by $O$ depends only on the parameters $a_{3}$ and $\bar{k}^{3}$ of the degree of freedom along the third axis. This is because the factorization of the function (62) guarantees the independence of the space degrees of freedom.

\section{Concluding remarks}

Resorting to the canonical quantization in radiation gauge we constructed in premier the quantum theory of the plane wave packets of the Maxwell free field propagating in the de Sitter expanding universe, studying how these are detected by observers staying at rest in the origins of their proper frames with physical coordinates. We have shown that the observer's detector filters a one-dimensional wave packet propagating accelerated, dispersing exponentially and having a maximum intensity affected by an exponential decay during propagation. This decay leads to the rule (59) allowing us to estimate the maximum intensity of the prepared wave packet. We must specify that our preliminary calculations indicate that, applying the same method, we find that this relation holds in any spatially flat FLRW space-time.

It is remarkable that in this approach the horizon effects may be pointed out analytically as the wave packets prepared on observer's horizon remain static while these prepared beyond his horizon appear as being reflected (as in Fig. 3 ) such that these cannot be observed. This intuitive behavior is one of the advantages of the physical coordinates we use here. Note that these coordinates are suitable for studying the local relative motion rather than general cosmologies where the FLRW coordinates are preferred.

Concluding, we may say that the present paper completes the quantum theory of the Maxwell field in de Sitter expanding universe we proposed so far $[46,62]$. The next step may 
be the generalization of this approach to the spatially flat FLRW space-times which have conformal frames where we can take over the results of the relativistic electrodynamics in Minkowski space-time. Moreover, in these manifolds we can introduce physical coordinates regardless the difficulties related to possible evolving observer's horizons. The only impediment seems to be the momentum-dependent phase of the fundamental plane wave solutions, which must be fixed in each particular case separately. Thus we may have the perspective of a complete quantum theory of the Maxwell field propagating in various expanding universes of interest in the actual cosmology.

Data Availability Statement This manuscript has no associated data or the data will not be deposited. [Authors' comment: This is a theoretical study and no experimental data has been listed.]

Open Access This article is licensed under a Creative Commons Attribution 4.0 International License, which permits use, sharing, adaptation, distribution and reproduction in any medium or format, as long as you give appropriate credit to the original author(s) and the source, provide a link to the Creative Commons licence, and indicate if changes were made. The images or other third party material in this article are included in the article's Creative Commons licence, unless indicated otherwise in a credit line to the material. If material is not included in the article's Creative Commons licence and your intended use is not permitted by statutory regulation or exceeds the permitted use, you will need to obtain permission directly from the copyright holder. To view a copy of this licence, visit http://creativecomm ons.org/licenses/by/4.0/.

Funded by SCOAP ${ }^{3}$.

\section{References}

1. J.L. Synge, Mon. Not. R. Astron. Soc. 131, 463 (1966)

2. V. Perlick, O.Yu. Tsupko, G.S. Bisnovatyi-Kogan, Phys. Rev. D 97, 104062 (2018)

3. G.S. Bisnovatyi-Kogan, O.Yu. Tsupko, Phys. Rev. D 98, 084020 (2018)

4. J.T. Firouzjaee, A. Allahyari, Eur. Phys. J. C 79, 1140 (2019)

5. Z. Chang, Q.-H. Zhu, JCAP 06, 055 (2020)

6. S. Vagnozzi, C. Bambi, L. Visinelli, Class. Quantum Gravity 10, $1088(2020)$

7. O.Yu. Tsupko G.S. Bisnovatyi-Kogan, Int. J. Mod. Phys. D 29, 2050062 (2020)

8. J.M. Bardeen, Proceedings, Ecole d'Et de Physique Thorique: Les Astres Occlus (Les Houches, France, 1973)

9. H. Falcke, F. Melia, E. Agol, Astrophys. J. Lett. 528, L13 (1999)

10. A. Grenzebach, V. Perlick, C. Lämmerzahl, Phys. Rev. D 89, 124004 (2014)

11. Z. Stuchlik, D. Charbulák, J. Schee, Eur. Phys. J. C 78, 180 (2018)

12. P.-C. Li, M. Guo, B. Chen, Phys. Rev. D 101, 084041 (2020)

13. Z. Chang, Q.-H. Zhu, Phys. Rev. D 101, 084029 (2020)

14. F. Atamurotov, A. Abdujabbarov, B. Ahmedov, Phys. Rev. D 88 , 064004 (2013)

15. Z. Li, C. Bambi, J. Cosmol. Astropart. Phys. 01, 041 (2014)

16. A. Grenzebach, V. Perlick, C. Lämmerzahl, Int. J. Mod. Phys. D 24, 1542024 (2015)

17. P.V.P. Cunha, C.A.R. Herdeiro, E. Radu, H.F. Runarsson, Phys. Rev. Lett. 115, 211102 (2015)
18. A. Abdujabbarov, M. Amir, B. Ahmedov, S.G. Ghosh, Phys. Rev. D 93, 104004 (2016)

19. C. Bambi, K. Freese, S. Vagnozzi, L. Visinelli, Phys. Rev. D 100, 044057 (2019)

20. S. Vagnozzi, L. Visinelli, Phys. Rev. D 100, 024020 (2019)

21. K. Jusu, M. Jamil, P. Salucci, T. Zhu, S. Haroon, Phys. Rev. D 100, 044012 (2019)

22. P.V.P. Cunha, C.A.R. Herdeiro, E. Radu, Universe 5, 220 (2019)

23. S. Haroon, M. Jamil, K. Jusu, K. Lin, R.B. Mann, Phys. Rev. D 99, 044015 (2019)

24. S.-W. Wei, Y.-X. Liu, R.B. Mann, Phys. Rev. D 99, 041303 (2019)

25. N. Bar, K. Blum, T. Lacroix, P. Panci, J. Cosmol. Astropart. Phys. 07, 045 (2019)

26. H. Davoudiasl, P.B. Denton, Phys. Rev. Lett. 123, 021102 (2019)

27. I. Banerjee, S. Chakraborty, S. SenGupta, Phys. Rev. D 101, 041301 (2020)

28. R. Roy, U.A. Yajnik, Phys. Lett. B 803, 135284 (2020)

29. R. Roy, S. Chakrabarti, Phys. Rev. D 102, 024059 (2020)

30. C. Li, S.-F. Yan, L. Xue, X. Ren, Y.-F. Cai, D.A. Easson, Y.-F. Yuan, H. Zhao, Phys. Rev. Res. 2, 023164 (2020)

31. E.R. Harrison, Cosmology: The Science of the Universe (Cambridge Univ. Press, New York, 1981)

32. E. Harrison, Astrophys. J. 403, 28 (1993)

33. G.E. Lemaître, Ann. Soc. Sci. de Bruxelles 47A, 49 (1927)

34. G.E. Lemaître, MNRAS 91, 483 (1931)

35. E. Hubble, Proc. Natl. Acad. Sci. 15, 168 (1929)

36. I.I. Cotăescu, Eur. Phys. J. C 77, 485 (2017)

37. I.I. Cotăescu, Eur. Phys. J. C 78, 95 (2018)

38. I.I. Cotăescu, Mod. Phys. Lett. A 36, 2150022 (2021)

39. I.I. Cotăescu, Eur. Phys. J. C 81, 32 (2021)

40. I.I. Cotăescu, accepted for publication in MPLA. arXiv:2101.02019

41. A. Higuchi, L.Y. Cheong, Phys. Rev. D 78, 084031 (2008)

42. A. Higuchi, L.Y. Cheong, J.R. Nicholas, Phys. Rev. D 80, 107502 (2009)

43. S. Faci, E. Huguet, J. Queva, J. Renaud, Phys. Rev. D 80, 124005 (2009)

44. S. Faci, E. Huguet, J. Renaud, Phys. Rev. D 84, 124050 (2011)

45. D. Bini, G. Esposito, R.V. Montaquila, GRG 42, 51 (2010)

46. I.I. Cotăescu, C. Crucean, Progr. Theor. Phys. 124, 1 (2010)

47. A.A. Saharin, A.S. Kotanjian, H.A. Nersisyan, Phys. Lett. B $\mathbf{7 2 8}$, $141(2014)$

48. N.D. Birrell, P.C. Davies, L.H. Ford, J. Phys. A 13, 961 (1980)

49. K.-H. Lotze, Class. Quantum Gravity 2, 351, 363 (1985)

50. K.-H. Lotze, Class. Quantum Gravity 4, 1437 (1987)

51. K.-H. Lotze, Class. Quantum Gravity 5, 595 (1988)

52. K.-H. Lotze, Nucl. Phys. B 312, 673 (1989)

53. I.L. Buchbinder, E.S. Fradkin, D.M. Gitman, Forstchr. Phys. 29, 187 (1981)

54. I.L. Buchbinder, L.I. Tsaregorodtsev, Int. J. Mod. Phys A 7, 2055 (1992)

55. L.I. Tsaregorodtsev, Russ. Phys. J. 41, 1028 (1989)

56. J. Audretsch, P. Spangehl, Class. Quantum Gravity 2, 733 (1985)

57. J. Audretsch, P. Spangehl, Phys. Rev. D 33, 997 (1986)

58. I.I. Cotăescu, C. Crucean, Phys. Rev. D 87, 044016 (2013)

59. I.I. Cotăescu, J. Phys. A Math. Gen. 33, 9177 (2000)

60. I.I. Cotăescu, Phys. Rev. D 65, 084008 (2002)

61. I.I. Cotăescu, GRG 43, 1639 (2011)

62. I.I. Cotaescu, EPJC. arXiv:2102.12100

63. S. Drell, J.D. Bjorken, Relativistic Quantum Fields (Me Graw-Hill Book Co., New York, 1965)

64. P. Painlevé, C. R. Acad. Sci. (Paris) 173, 677 (1921)

65. G. Pascu, arXiv: 1211.2363

66. I.I. Cotăescu, Mod. Phys. Lett. A 22, 2965 (2007)

67. I.I. Cotăescu, C. Crucean, A. Pop, Int. J. Mod. Phys. A 23, 2563 (2008) 
68. I.I. Cotăescu, Int. J. Mod. Phys. A 35, 2030019 (2020)

69. N.D. Birrell, P.C.W. Davies, Quantum Fields in Curved Space (Cambridge University Press, Cambridge, 1982)

70. S. Weinberg, The Quantum Theory of Fields (Univ. Press, Cambridge, 1995)

71. K. Bleuler, Helv. Phys. Acta (in German) 23, 567 (1950)

72. S. Gupta, Proc. Phys. Soc. 63A, 681 (1950)

73. I.I. Cotăescu, Eur. Phys. J. C 79, 696 (2019)

74. I.I. Cotăescu, Eur. Phys. J. C 80, 621 (2020)
75. I.I. Cotăescu, Eur. Phys. J. C 80, 535 (2020)

76. I.I. Cotăescu, Chin. Phys. C 45 (2021)

77. A. Messiah, Quantum Mechanics (Dover, New York, 1999)

78. I.I. Cotăescu, Mod. Phys. Lett. A 32, 1750223 (2017)

79. I.I. Cotăescu, arXiv:2102.03211

80. F.W.J. Olver, D.W. Lozier, R.F. Boisvert, C.W. Clark, NIST Handbook of Mathematical Functions (Cambridge University Press, Cambridge, 2010) 